Monatsschrift f. Geburtshülfe u. Gynäkologie 1925;71:116-120

\title{
III. Literaturverzeichnis
}

\section{Geburtshülfe.}

Bartholomew, Klinische Darstellung und Bedeutung der kindlichen Herz-

töne während der Geburt. Amer. Journ. obst. gyn. X. 1. S. 89. Fitz-Gibbon und Bigger, Klinische und bakteriologische Untersuchungen

des Puerpalfiebers im Rotundahospital in Dublin. Brit. Emp. Journ.

Sommer N. 1925. S. 298. Gümor, Smith, Gatenby, Primäre Ovarialsehwangerscliaft,

msbesondere ihre

Beziehungen zum Corpus luteum. Brit. Emp. Journ. Sommer X. 1925.

S. 339. Henneberg und Bíckel, Diabetes, Schwangersehaft. Insuline. Gyn. obst.

XII. 1. S. 72. Hofbancr, Die Funktion der Hofbauerzellen der Chorionpotten, in bezug auf akute Infektion und Syphilis. Amer. J. obst. gyn. X. 1. S. 1. Jarcho, Die Kiellanclzange und ihre Anwendung. Amer. J. obst. gyn.

XI. 1. S. 35. McCaffrey, Verlängerte und verkürzte Schwangerschaftsperioden vom ärztlichen und gerichtsärztlichen Standpunkt. Amer. J. obst. gyn.

X. 1. S. 107. McCullagh, Inversio uteri. 3 Fälle und Analyse von 233 neuen Beobachtungen. Brit. Emp. Journ. Sommer N. 1925. S. 280. Michael, Der Bandlsche Ring als Geburtshindernis. 3 Fälle. Amer. J.

obst. gyn. X. 1. S. Ill. Petersen, Geburtshilfl. Paralyse des Xerv. radialis. Gyn. obst. XII. 1. S. 68. Proshek, Fall von Sympusdipus. Anatomische Studie. Amer. J. obst.

gyn. X. 1. S. 15. Schickele, Die Rolle der Plazenta im mütterlichen Organismus. Gynèc· obstétr. XII. 1. S. 1. Schulze, Margarete, Die Bedeutung des Mekoniumabganges während der Geburt. Amer. J. obst. gyn. X. 1. S. 83. Vignes, Verhaltung der Frucht im Uterus. Progrès medical. 19. V. 25. Whitridge Williams, Weitere Beobachtungen von vorzeitiger Lösung der normal inserierten Plazenta. Brit. Emp. Journ. Sommer X. 1925.

S. 259.

Literaturverzeichnis.

117

Gynäkologîe.

Berkeley, Über die Technik der Radikaloperation des Kollumkrebses. Brit.

Empire Journ. Sommernummer 1925. S. 346. Bonnet, Über die Diagnostik der peritonealen Uberseliwemmung und die

akute Appendizitis. Gyn. obst. XII. 1. S. 36. Douay, Tubare Sterilität. Gyn. obst. XII, 1. S. 80. Fothergill, Fehlen der Scheide. Hämatometra, Hämatosalpinx und Teerzyste. Brit. Empire J., Sommernummer 1925. S. 336. Gelliiorn, Einige strittige Punkte der Uteruskurettage. Amer. J. obst.

gyn. X. 1. S. 101. Halperin, Schwerer portoperativer Lungenkollaps. Surg. gyn. obst. XLI. 1. S. 53. Harris, Teratoma ovar. carcinomatosum mit Pubertas praecox und vorzeitiger somatischer Entwicklung. Surg. gyn. obst. Aug. 1925. S. 191. Hurd, Eine Reihe von. Todesfällen gynäkolog $1 / 8$ cher Patienten. Amer. J. 
obst. gyn. X. 1. S. 120. Ivens, Endometriomyom der hinteren Uteruswand. Brit. Empire Journ. Sommernummer 1925. S. 344. Kammerer, Ungewöhnliche Erfahrung mit der Xephrektomie bei Nephr.

suppurativa. Surg. gyn. obst. XLI. 1. S. 70. Lamson, Abdominale Verwachsungen. Surg. gyn. obst. Aug. 1925. S. 230. Lavake, Diathermie mit Metalleiektrode, vielleicht ein

Beihilfinstrument

für die Behandlung weiblicher Gonorrhöe. Surg. gyn. obst. XLI. 1.

S. 31. Mundell, 4 Jahre Erfahrung mit Radium bei der Behandlung gynäkologischer Verhältnisse. Amer. J. obst. gyn. X. 1. S. 70. Ott, J., 2 Fälle von primärem Klitòriskarzinom. La clinica ost. XXVII. 6.

S. 266. v. Ott, Ein Versuch, die Finger Minstlich zu verlängern. Ztrbl. f. Gyn. Nr. 19.

S. 1035. Ottow, Die klinischs Bedeutung der Technik der Eileiterdurchblasung.

D. med. Woch. Nr. 19. S. 783. Parrnenteo, Zystologische Untersuchung des Harnsalzes. Beihilfe der

Untersuchung der Diagnose genito-urinaler Tumoren. Surg. gyn. obst.

April 1925. S. 531. Pasch, Kombinierte spezifische und imspezifiscbe Gonorrhöebehandlung beiFrauenmit Sublimat-Olobintin. Dtsch. med. Woch. Nr. 15. S. 607. Penkert, Zur Antefixation des retroflektierten Uterus bei Laparatomien.

Ztrbl. f. Gyn. Nr. 20. S. 1086. Petzol, Kritisches Sammelreferat über Gonorrhöebehandlung mit Silber-

präparaten. Dtsch. med. Woch. Nr. 13. S. 541. Polak und Phelan, Zervixkrebsbehandlung.

Radium oder Operation?

Sammelreferat. Amer. J. obst. gyn. X. 1. S. 140. Popper, Regulative Reiztherapie der Staphylokokkenerkrankungen und

der Gonorrhöe. Klin. Woch. Nr. 22. S. 1063. von der Porten, Narzylen in der Privatpraxis. M. med. W. Nr. 25. S. 1027. Pösch, Über den Nachweis von Hämosiclerin im Endometrium. Arch. f.

Gyn. Bd. 123. S. 671. Reel, Die Gefahr zervikaler Erosionen. Amer. J. obst. gyn. X. 1. S. 94. 118

Literaturverzeichnis.

Reid, Ein peritonealer Sack mit Dünndarm. Bemerkung zu der Knickung des Ileums. Surg. gyn. obst. XI. 3. S. 400. Ricard und Lecomte, Akute peritoneale Formen der adnexiellen Bazillose.

Gyn. obst, XII. 1. S. 48. Robinson, Kritik der Histogenese heterogener endometrialer Wucherungen.

Surg. gyn. obst. XL·I. 1. S. 36. Roedelius, Über Blasenverletzungen bei Eröffnung von Douglasabszessen.

D. med. W. Xr. 28. S. 1151. Roeder, Eine modifizierte Xabel-Herniotomie nach Mayer. Surg. gyn. obst.

Mai 1925. S. 707. Rother, Über die Veränderung des Blutagars durch Streptokokken. I). med. W. Xr. 25. S. 1031. Rother, Über Scheidendesinfektion. Munch, med. Woch. Xr. 23. S. 937.

Ders., Der Bacillus vaginae Döderlein und der Abbau des Glykogens im

Genitaltraktus. Ztrbl. f. Gyn. Nr. 25. S. 1357. Rühl, Regelmäßigkeit im Wechsel der Ovarialfunktion. Arch. f. Gyn.

Bd. 124. Heft 1. S. 1. Runge, Die Gynäkologie des praktischen Arztes. 2. Auflage. Berlin und 
Wien 1925. Urban \& Schwarzenberg. Sachs, Zur Behandlung der chronischen Gonorrhöe der Frau. (Gleich-

zeitige Frischvakzine und Lokaltherapie.) Z. f. G. Xr. 12. S. 648. Salomons, Ergebnis einer

Rundfrage über die subperitoneale Gilliam-

Operation innerhalb der letzten 6 Jahre. British Empire Journ.

Sommernummer 1925. S. 330. Schapiro, Gonorrhöebehandlung mit ïargesin. Med. Klin. Xr. 23.

S. 859. Schi††mann und Szamek, Zur Kenntnis und Genese entzündlich-eitriger

Veränderungen der Niere nach Ureterscheidenfisteln. W. kl. W. Xr. 23.

S. 644 (Festschrift). Schmidt, Helmut, Zur Xarzylennarkose. Munch, med. Klin. Xr. 21. S. 841.

Schmidt, Entzünclhche oder blastoinatöse Tumorenentwicklung in der

weiblichen Harnröhre ? Ztschr. f. Geb. u. Gyn. Bd. 88. Heft 3. S. 563. Schneider, G. H.,

Vereiterung einer Hämatokele nach Extrauteringravidität

durch Paratyphusinfektion. Ztrbl. f. Gyn. Nr. 26. S. 1443. Schneider, Georg Heinr., Die

Voraussetzung und Technik für eine gefahrlose

Bluttransfusion und Untersuchungen über die Isohämagglutination.

Arch. f. Gyn. Bel. 124. Heft 1. S. 213. Schneider, Paul, Über Todesfälle durch Bluttransfusion und deren Ver-

meidung. Ztrbl. f. Gyn. Xr. 17. S. 900. Schneider, Über das Verfahren der Transfusion. Z. f. G. Nr. 13. S. 703. Schoenholz, Das Problem der temporären Strahlenkastration der Frau.

Munch, med. Woch. Nr. 22. S. 886. Schoog, Mitteilungen über das Uteruskarzinom in der

Kölner Frauenklinik

während der Jahre 1906-1925. Ztrbl. f. Gyn. Nr. 27. S. 1492. Schugt, Ein Fall von

Uterusperforation mit schwerer Verletzung und

nachfolgender ausgedehnter B, esektion des Dünndarms. Z. f. G.

Xr. 15. S. 814. Schumacher, Klinische und path.-anatom. Beobachtungen an Frauen, denen

Blut nach der Methode von Oehlecker transfundiert wurcle. Ztschr.

f. Gyn. u. Geb. Bd. 88. Heft 3. S. 591.

Literaturverzeichnis.

119

Schumacher, Zur Frage der Auswahl unter den Spendern für die intra-

venöse Transfusion größerer Blutmengen. Arch. f. Gyn. Bd. 123.

S. 569. Schwab, Die Vakzinetherapie der weiblichen Gonorrhöe. Munch, med. Woch.

Nr. 19. S. 767. Schwarz, Klinische Beobachtungen und experimentelle Untersuchungen

über die Resorptionsfähigkeit der Harnröhren- undBlasenschleimhaut

beim Menschen (Bemerkungen zur gleichnamigen Arbeit von Schönfeld

und Midler in N $\gamma$. 8). Munch, med. Woch. Nr. 18. S. 737. Schwartz, L., Stieldrehung von

Eierstockskrebsen. Amer. J. obst. gyn.

IX. 3. S. 375. Seeland, Zur Frage der Behandlung der postoperativen Harnverhaltung

mit Kalium. Munch, med. Woch. Nr. 17. S. 688. Seidler, Zystadenoma cler großen Schamlippen embryonalen CJrsprungs.

Ztrbl. f. Gyn. Nr. 28. S. 1569. Seidler, Die Lumbo-Sakralanästhesie bei gynäkologischen Operationen.

Ztrbl. f. Gyn. Nr. 19. S. 1040. Sélilzky, Der Eklampsismus. Gynéc. obstét. XI. 6. S. 428. Send, Omnadin in der Abdominalchirurgie. D. med. Woch. Nr. 22. S. 911. Siegert, Friedrich, Die

Funktion der glatten Muskulatur und ihr Einfluß 
auf die Schmerzentstehung. Arch. f. Gyn. Bd. 123. S. 774. Siemens, Gibt es eine Hämophilie beim Weibe ? Arch, f. Gyn. Bd. 124.

Heft 2. S. 374. Simon, Die subjektiven Beschwerden bei Anlegung eines Pneumoperitoneums. Med. Klin. Nr. 16. S. 589. Simonds und Gowen, Fetus amorphus. Surg. gyn. obst. August 1925.

S. 171. Sommer, Das Hämogramm in der Gynäkologie. Ztschr. f. Geb. u. Gyn.

Bel. 88. Heft 3. S. 658. Soper, Tatsachen und Ansichten auf Grund von Laboratoriumversuchen zu praktischer Arbeit in den Krebsfragen. Surg. gyn. obst. XI. 3.

S. 334. Soresi, Künstlicher Nabelafter. Surg. gyn. obst. XLI 1. S. 103. Southern Surgical Society. XXXVI. Bd. Verhandlungen (11.-13. XII. 23).

Herausgegeben Royster, Raleigh N. C. Philadelphia, Dornan. Soutlar, Eine neue Form von einer Ligaturnadel. Surg. gyn. obst. April 25.

S. 565. Sserdjukoff, Zur Frage der iiikretorischen Störungen der Ovarien und epileptiforme Anfälle als Ausdruck eines pluriglandulären Syndrom.

Arch. f. Gyn. Bd. 124. Heft 1. S. 284. Stamm, Über Extrauteringravidität. Ztschr. f. Geb. u. Gyn. Bd. 88. Heft 3.

S. 553. Straßmann, Ersatz einer Schrumpfblase durch Transposition des Sromanum.

Ztrbl. f. Gyn. Nr. 21. S. 1122. Strauß, Über Krebs und Krebsbehandlung (Sammelreferat). Med. Klin.

Nr. 15. S. 554. Nr. 16. S. 596. Nr. 17. S. 633. Stabler und Haeuber, Die heterope, endometroide Epithelwucherung im

weiblichen Genitalapparat, insbesondere im Ovarium. Arch. f. Gyn.

Bd. 124. Heft 2. S. 305.

120

Literaturverzeielmis. - Personalien.

Szegö, Über Hauterkrankungen bei Störungen der Ovarialfunktion. Zti·bl.

' f. Gyn. Nr. 19. S. 1018. Tagliaferro, Hysterektomie bei scbwerer Metrorrhagie bei zweifelhafter

Diagnose. La clinica ostetr. XXVII. 3. S. 114. Tateyama, Über einen Fall von intraperitonealer Exstirpation eines Ureter -

steines. Ztrbl. f. Gyn. Nr. 21. S. 1134. Turenne, Odem und Hypertrophie der Zervix uteri

während des Puerperium.

Surg. gyn. obst. August 1925. S. 156. Turolt, Beitrag zur operativen Therapie der

Tubargravidität. Ztrbl. f. Gyn.

Nr. 27. S. 1467. Vignes, Thyreoidea-Suprarenalis-Hypophyse während der Schwanger-

schaft. Gaz. medic, du centre. 15. IV. 1925. Vogí, Über die Zusammenhänge zwischen der

Gonorrhöe und den gynä-

kologischen Symptomen des Fluors, der Blutungen und der Schrnerzen.

K1. W. Nr. 27. S. 1316. Vogt, Über den Geruch bei der klinischen Diagnose von intraperitonealen

Blutergüssen. Med. Klin. Nr. 22. S. 804. Vorlaender, Heilungsversuche am experimentellen

Krebs. Klin. Woch.Nr. 23.

S. 1120. Walthard, Zur Pathogenese psychisch bedingter Symptomenkomplexe im weiblichen Genitale. (Psychisch bedingte Genitalneurosen.) Arch.

f. Gyn. Bd. 124. Heft 2. s' 381. WeibeL·, Lassen sich die Behandlungserfo $1 / 8$ e bei Carcinoma colli uteri ver- 
bessern? W. kl. W. Nr. 23. S. 666 (Festschrift). Weißenberg, Über L/nfruchtbarkeit. Z. f. G. Nr. 13. S. 706. Wermbter, Statistischer Beitrag über Thrombose und Limgenembolie im Iiinblick auf die TrendelenburgHclie O])eration. Ztrbl. f. Gyn. Nr. 28.

S. 1560. Werner, Zur Therapie des Fluors. W. k1. W. Nr. 23. S. 660 (Festschrift). Wieloch, Beitrag zur Entstehung heterotoper L"terusschleimhaut und De-

zidua. Arch. f. Gyn. Bd. 124. S. 53. Wolff, Klavipurin in der gynäkologischen Praxis. D. med. Woch. Nr. 18.

S. 774. Zaraíe, Neue Technik der subkutanen Symphyseotomie. Gynéc. obstét.

XL 6. S. 401. Zimmermann, Frauenheilkunde. (i'ortschritte der gosamten Medizin.)

D. med. Woch. Nr. 23. S. 952. Nr. 24. S. 994.

Strahle $\pi$ therapie.

Mc Arthur, Schädigender Einfluß der ultravioletten Strahlen auf alte X-Strahl-Verbrennungen.

Surg. gyn. obst. XLI. 1. S. 97.

Personalien.

Privatdozent Dr. W. Schmitt, Oberarzt der Universitäts-Frauenklinik in Würzburg, erhielt den Titel a.o. Professor.

Professor Hermann .FmфrtcZ-Fraiikfurt a. M. ist Anfang September ge-storben. Die Monatsschrift wird die Lebensarbeit ihres verehrten Mitarbeiters- $\pi$ nd verdienten Kollegen in einem der nächsten Hefte $\Lambda$ vürdigen. 